\title{
Channel estimation based on the PSS- MUSIC for millimeter-wave MIMO systems equipped with co-prime arrays
}

\author{
Shufeng Li $i^{*} \mathbb{D}$, Guangjing Cao ${ }^{1}$, Libiao $\mathrm{Jin}^{1}$ and Hongda Wu²
}

\begin{abstract}
The millimeter-wave channel estimation problem can be solved by estimating the path directions and the path gains. The previous schemes are nearly all based on the uniform linear arrays (ULAs). However, compared with the ULAs, the co-prime array can use fewer array elements to realize larger array aperture, which is beneficial to improve the performance of arrival direction estimation contributing to the estimation performance of angle of arrival (AOA) and angle of departures (AOD). Encouraged by the property of co-prime arrays, a novel channel estimation scheme is proposed for two-dimensional (2-d) co-prime arrays. For each path direction, multiple peaks are generated in the spatial spectrum of each subarray and by selecting over any limited sector to search for an arbitrary peak, which can recover the rest peaks. Then by comparing the peaks of the two subarrays, the common peaks are the correct path direction. Compared with the total-spectrum search (TSS) method, this method effectively reduces the complexity. Simulation results show that the proposed algorithm can reduce the computational complexity and maintain accurate channel estimation.
\end{abstract}

Keywords: Millimeter-wave MIMO communications, Two-dimensional channel estimation, Co-prime arrays, Uniform arrays

\section{Introduction}

Massive MIMO (multiple-input and multiple-output) technologies have been widely used in current $5 \mathrm{G}$ wireless communication systems [1-5]. The millimeter wave (mm-wave) and massive MIMO are combined to obtain enough signal power and improve the transmission distance. The traditional channel estimation techniques for MIMO systems fail to characterize the spatial sparsity of the mm-wave channel; the problem of mm-wave channel estimation can be turned into estimating the path directions and gains rather than estimating the MIMO channel matrix $[6,7]$.

Before discussing the non-uniform arrays, the channel estimation methods based on uniform linear array systems are briefly reviewed. Due to the spatial sparsity in $\mathrm{mm}$ wave channel, traditional channel model based on rich

\footnotetext{
* Correspondence: shufeng_2004@163.com

${ }^{1}$ School of Information and Telecommunication Engineering, Communication University of China, Beijing 100024, People's Republic of China

Full list of author information is available at the end of the article
}

scattering is not practical. Taking advantage of the sparse characteristics of $\mathrm{mm}$-wave signals which mean that there is only a small amount of important information, the first class of channel estimation method represented by beam training method has been introduced [8, 9]. In addition, in order to reduce the signal pilot training cost, the accuracy of angle estimation is improved by combining compression sensing $(C S)$ with beam training scheme $[10,11]$. It is worth mentioning that this method also compares the amplitude of auxiliary beam pair to improve the estimation accuracy. In addition, the orthogonal matching pursuit (OMP) algorithm [12-14] is used based on mm-wave MIMO channel systems. If there is an assumption that the path direction is distributed discretely in the angle domain where we need to emphasize is that the actual AOA and AOD are continuously distributed in the angle domain $[15,16]$ can utilize the angular channel sparsity to solve the problem of training overhead when estimating the channel information. Some scholars have proposed an idea based on iterative weight (IR) to deal with the problem of resolution distortion caused by the on-grid angle 
estimation [17]. Deep learning is also applied to millimeter wave systems improve the performance of communication systems $[18,19]$.

In addition to the channel estimation scheme already mentioned, classical spatial spectrum estimation, especially MUSIC algorithm, has been used in mm-wave channel estimation for a long time. It was originally used to estimate AOA in [20]. Then, this method can be used to estimate both the departure and arrival angles in [21, 22], and among them, the [22] utilized the two-dimensional MUSIC. A two-dimensional (2-d) channel estimation scheme is proposed for the systems with uniform planar arrays (UPAs) in [23]. Channel estimation based on spatial spectrum estimation can theoretically improve the angular estimation accuracy, angular resolution, and other related parameter accuracy of spatial signals in the system processing bandwidth [24]. However, based on this method, matrix decomposition, spectral peak search, and multidimensional parameter optimization require a large number of complex multiplication operations, and the number of operations is proportional to the cube of the number of array elements [25]. Therefore, when the number of array elements is large, the computational complexity of the channel estimation algorithm is extremely high, which is not conducive to engineering implementation. In order to avoid angle ambiguity, the spacing of the equidistant ULA should be no more than half of the wavelength of the radiation, so communication systems based on the ULA can only achieve the expansion of the array aperture by increasing the number of array elements, which is limited to some extent [26]. In order to solve this problem, we can use the new array to reduce the number of array elements without guaranteeing the loss of the array aperture. In recent years, a new geometry of non-uniform linear arrays (ULAs), denoted as coprime arrays attract more attention, which can obtain large array aperture with fewer array elements [27]. These unique advantages of the co-prime arrays encourage researchers to conduct a series of related research. Co-prime MIMO radar is one of the typical applications in practice; however, there are too few studies on channel estimation in MIMO communication systems equipped with co-prime arrays, so next the application of co-prime arrays in DOA estimation will be introduced.

Due to the high computational complexity and low adaptability, several commonly used DOA estimation methods [28-30] can result in huge computational complexity if applied directly to the co-prime array. Therefore, some scholars have specifically proposed estimation algorithms for systems with co-prime arrays. The TSS-MUISC scheme using the entire spectral search first appeared in [31]. In this article, DOA is obtained by selecting common peaks in two separated arrays. It is not difficult to find that the search scope is so large leading to a sharp rise in the computational dimension. Considering the maximum peaks generated by each signal source are distributed uniformly in the period range, it has been proposed to change the original entire search to partial search which greatly reduces the number of searches. That is PSS-MUSIC [32] where for each DOA, multiple peaks are generated on the spatial spectrum of each subarray and by selecting any limited sector to search for an arbitrary peak to recover the other ones. This is followed by [33] which demonstrates a low-complexity DOA estimation method based on ESPRIT. In order to replace the MUSIC algorithm, it uses the ESPRIT algorithm to perform angle estimation. In this way, spectrum search is completely avoided to further reduce computational complexity. An improved Root-MUSIC-based DOA was proposed in [34], and the estimation accuracy has been improved. In this article, we will mainly improve the method in [31,32] to estimate the path direction.

In this paper, we propose a computationally efficient channel estimation scheme for 2-d co-prime arrays where the directions can be uniquely estimated by finding the common peaks of the two decomposed subarrays. It can substantially reduce the complexity while maintaining high resolution, as compared with the TSSMUSIC method.

The main contributions of this paper are as follows:

a) From the perspective of the array, the channel estimation accuracy is improved by employ a new array. The influence of the two arrays on the channel parameters is compared under the premise of the same algorithm, which proves the superiority of the co-prime array.

b) Encouraged by the advantages of the co-prime arrays, a novel channel estimation scheme is proposed for 2-d co-prime arrays. We have proved the superiority of the new algorithm theoretically by comparing the complexity analysis with the traditional method.

c) Change the signal-to-noise ratio and the number of snapshots separately, we can find the relationship between the mean square error and these two variables. The simulation results show that the proposed channel estimation scheme can reduce the computational complexity. When the time of spectral search are similar, the novel algorithm has better estimation performance.

To be more specific, the rest of the paper is organized as follows: Section 2 introduces the methodology. Section 3 describes the signal model of communication system with co-prime planar array. The next section describes the problem and clarifies the reason. Section 5 derives the proposed method. Section 6 discusses the performance of the proposed method and provides the simulation results. And Section 7 concludes the work. 
The specific meaning of the symbols in this article is shown below: $\mathbf{A}$ is a matrix, $\mathbf{a}$ is a vector and $\operatorname{diag}(\mathbf{A})$ is a vector formed by the diagonal elements of $\mathbf{A} \cdot \mathbf{A}^{\mathrm{T}}, \mathbf{A}^{*}$, and $\mathbf{A}^{H}$ are the transpose, the conjugate, and the conjugate transpose, respectively. The inverse and pseudo-inverse is represented by $\mathbf{A}^{-1}$ and $\mathbf{A}^{\dagger}$. $\|\mathbf{A}\|_{F}$ is its Frobenius norm.

\section{Methodology}

In this article, we first introduce existing channel estimation methods and finds that they are nearly all based on the communication systems equipped with ULAs. The research background and related methods are presented in Section 1. There are many factors that affect the performance of channel estimation. This article creatively considers improving the performance of the system from the perspective of array shape. Compared with the ULAs, the co-prime array can use fewer array elements to realize larger array aperture which can reduce the cost of the system and decrease the subsequent computational complexity. Moreover, because of the property of the co-prime array, we do not need to consider the problem of angular ambiguity caused by too large array element spacing. Thus, changing the structure of the array reasonably is beneficial to improve the performance of arrival direction estimation contributing to the estimation performance of angle of arrivals (AOA) and angle of departures (AOD). During the study of the system model, Fig. 2 shows the relationship between the co-prime array and the uniform array in detail. In the simulation comparison, in order to better verify the effectiveness of the algorithm, we use a complex twodimensional array model, that is, an L-shaped array consist of co-prime arrays. This array has two outstanding advantages: one is that it does not ignore the azimuth compared to the linear array, and the other is to use a coprime array to form the two parts of the L-shaped array.

Encouraged by the advantage of the co-prime arrays, a computationally efficient channel estimation scheme for 2- $\mathrm{d}$ co-prime arrays is presented where the AOA and
AOD can be uniquely estimated by finding the common peaks of the two decomposed subarrays. Compared with the total-spectrum search (TSS) method, the channel estimation scheme based on the PSS algorithm can search over a limited area which can effectively reduce the complexity and does not change the resolution level.

In order to verify the effectiveness of the algorithm, we have conducted a variety of experiments to obtain comparison results. First, we first explain why we choose a co-prime array. Experiments show that the same algorithm performs better than a uniform array when using a co-prime array. Then, the complexity is calculated and the superiority of the new algorithm is proved mathematically. Finally, the new channel estimation algorithm is compared with the TSS-MUSIC algorithm. The specific analysis can be found in Section 6 .

\section{System model}

In this paper, we focus on a mm-wave MIMO communication system equipped with the hybrid precoding [22] which has been shown in Fig. 1.

$N_{t}$ and $N_{r}$ represent the number of the antenna array at the transmitter and receiver. One end of the analog beamformer $\mathbf{F}_{\mathrm{RF}}$ is connected the transmitter, and the other end is connected to $M_{t} \mathrm{RF}$ chains. The receiver has the same hybrid structure, the ends of $\mathbf{W}_{\mathrm{RF}}$ are connected to receiver and $M_{r} \mathrm{RF}$ chains. In the practice systems, the number of antennas is greater than that of RF chains, such that $N_{t}>M_{t}$, while the $\mathbf{F}_{\mathrm{BB}}$ and $\mathbf{W}_{\mathrm{BB}}$ denote the digital precoders and combiners.

The signal obtained at the receiver can be expressed by the following formula

$$
\mathbf{Y}=\mathbf{W}^{H} \mathbf{H F s}+\mathbf{W}^{H} \mathbf{n}
$$

where $\mathbf{F}=\mathbf{F}_{\mathrm{RF}} \mathbf{F}_{\mathrm{BB}}$ and $\mathbf{W}=\mathbf{W}_{\mathrm{RF}} \mathbf{W}_{\mathrm{BB}}$ are the hybrid precoder and hybrid combiner, respectively. $\mathbf{s}$ is the vector of

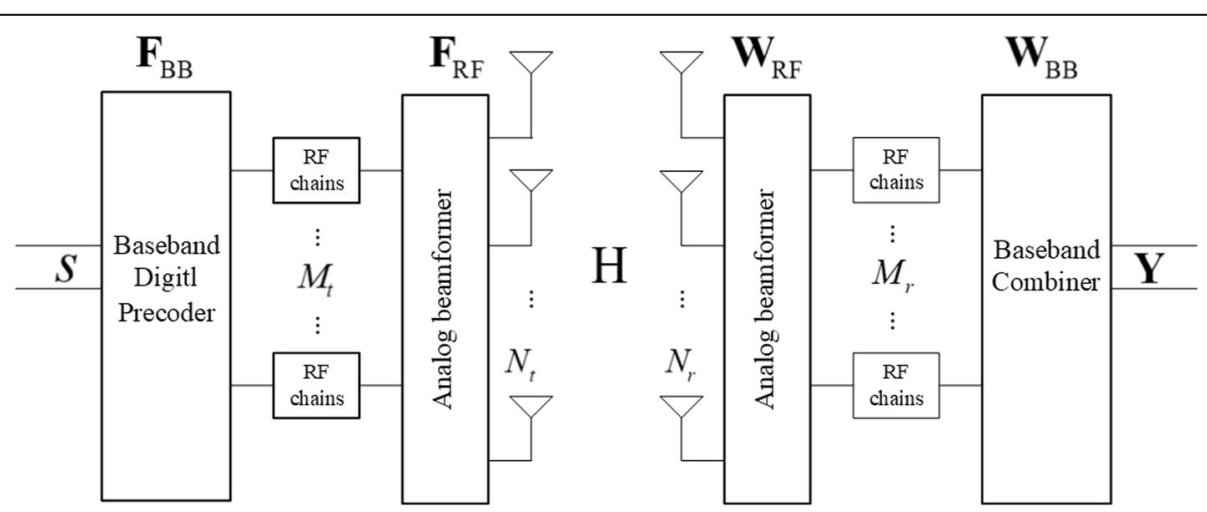

Fig. 1 Illustration of a hybrid analog/digital precoding and combing structure. The picture illustrates a mm-wave communication system equipped with hybrid analog/digital beamforming 
transmitted baseband signal, $\mathbf{H}$ is the channel matrix, and the noise $\mathbf{n}$ follows the distribution $\mathcal{C N}\left(0, \sigma_{n}^{2} I_{N}\right)$.

Supposing there are two uniform linear arrays which are consisting of $M$ and $N$ array elements where $M$ and $N$ are co-prime integers. As illustrated in Fig. 2a, the number of array elements in subarray above is $M$ where $N d$ is the inter-element spacing. Similarly, the number of array elements in subarray below is $N$ where $M d$ is the interelement spacing. Figure $2 \mathrm{~b}$ shows a co-prime array model setting $M=4$ and $N=3$ where the simple values are set for a more intuitive introduction. The $d$ is usually set as half wavelength $\lambda / 2$. The number of array elements in the entire array is $M+N-1$ because the first element positions of the two subarrays are coincident.

Then, introducing a $x-y$ plane L-shaped array which consists of two co-prime subarrays shown like Fig. 3. The L-shaped array has $M+N-1$-element arranged along the $X$-axis and an $M+N-1$-element arranged along the $Y$ axis. The spacing between adjacent elements of two subarrays is $M d$ or $N d$, respectively. The first element of the two subarrays serves as a common reference point.

Defining the elevation and azimuth by $\theta$ and $\phi$, then the channel model can be expressed as Eq. (2).

$$
\begin{aligned}
\mathbf{H}(q) & =\left[\begin{array}{l}
\mathbf{A}_{R x}\left(\theta_{r}, \phi_{r}\right) \\
\mathbf{A}_{R y}\left(\theta_{r}, \phi_{r}\right)
\end{array}\right] \boldsymbol{\Lambda}_{G}(q)\left[\begin{array}{l}
\mathbf{A}_{T x}\left(\theta_{t}, \phi_{t}\right) \\
\mathbf{A}_{T y}\left(\theta_{t}, \phi_{t}\right)
\end{array}\right]^{\mathrm{T}} \\
& =\mathbf{A}_{R}\left(\theta_{r}, \phi_{r}\right) \boldsymbol{\Lambda}_{G}(q) \mathbf{A}_{T}\left(\theta_{t}, \phi\right)
\end{aligned}
$$

where the number of paths is $L, \boldsymbol{\Lambda}_{G}(q)=\operatorname{diag}\left\{g_{1}(q)\right.$, $\left.\cdots g_{L}(q)\right\}, \mathbf{A}_{R}=\left[\mathbf{a}_{r}\left(\theta_{r 1}, \phi_{r 1}\right), \cdots, \mathbf{a}_{r}\left(\theta_{r L}, \phi_{r L}\right)\right]$, and $\mathbf{A}_{T}=\left[\mathbf{a}_{t}\left(\theta_{t 1}\right.\right.$, $\left.\left.\phi_{t 1}\right), \cdots, \mathbf{a}_{t}\left(\theta_{t L}, \phi_{t L}\right)\right]$.

Defining $\alpha_{r x}=e^{-j 2 \pi d_{x} \cos \phi_{r x} \sin \theta_{r x} / \lambda}, \alpha_{r y}=e^{-j 2 \pi d_{y} \sin \phi_{r y} \sin \theta_{r y} / \lambda}$, $\beta_{r x}=e^{-j 2 \pi d_{x} \cos \phi_{t x} \sin \theta_{t x} / \lambda}$, and $\beta_{r y}=e^{-j 2 \pi d_{y} \sin \phi_{t y} \sin \theta_{t y} / \lambda}$, we have

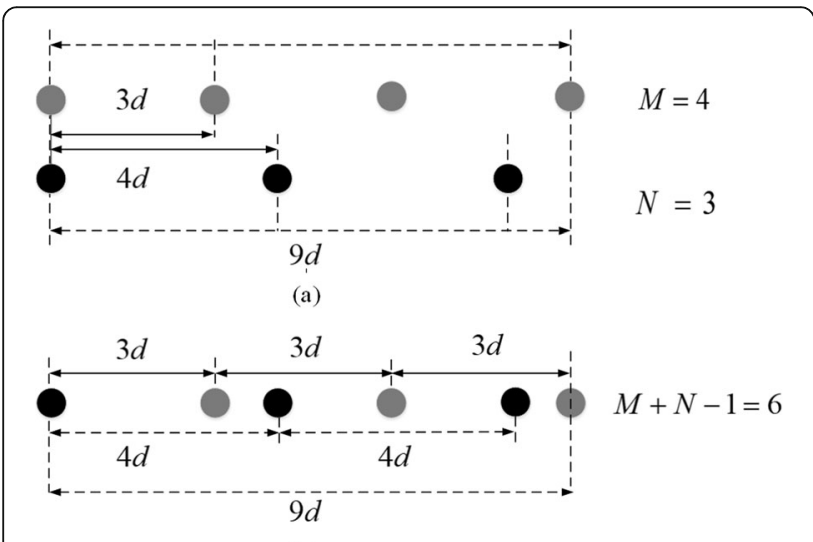

(b)

Fig. 2 Co-prime arrays. a Two uniform linear arrays. b Co-prime array formed by two uniform arrays. This picture gives an example of the co-prime arrays when $M=4$ and $N=3$ where the nonuniform array can be decomposed into two uniform linear arrays

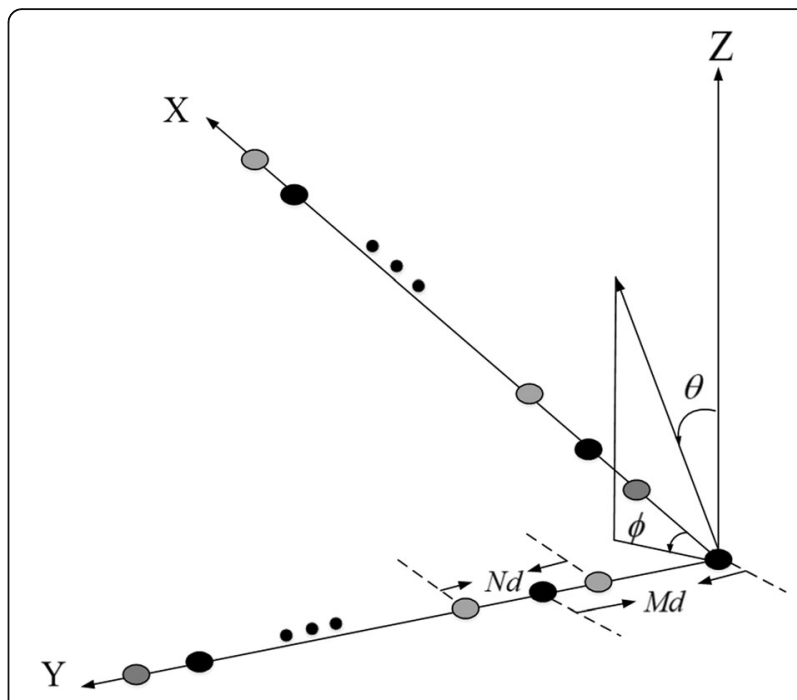

Fig. 3 The L-shaped array consists of co-prime arrays. It shows a plane L-shaped array consists of two co-prime subarrays where an $M+N-1$-element arranged along the $X$-axis and an $M+N-1$ element arranged along the $Y$-axis

$$
\begin{aligned}
& \mathbf{a}_{r}=\left[1, \alpha_{r x}, \cdots, \alpha_{r x}{ }^{N_{L x}} ; \alpha_{r y}, \cdots, \alpha_{r y}{ }^{N_{L y}}\right]^{T} \\
& \mathbf{a}_{t}=\left[1, \beta_{r x}, \cdots, \beta_{r x}{ }^{N_{L x}} ; \beta_{r y}, \cdots, \beta_{r y}{ }^{N_{L y}}\right]^{T}
\end{aligned}
$$

The channel direction and path have different fading scale where the former belongs to large scale fading and the latter belongs to small scale fading. The path directions will not change during a frame when the receiver can obtain $N_{b}$ time-fading block information which can be utilized to estimate the path directions. The gain of the path can be obtained according to the path directions. Based on the above theory, we can see the importance of estimating AOA and AOD.

\section{Problem formation}

\subsection{The impact of the arrays}

In the above channel estimation algorithm, the estimation of the angle parameter is a very important part of it, so we will analyze the effect of the antenna array structure on the direction estimation. It is worth noting that in the existing DOA estimation algorithm, uniform linear array is the most commonly used array structure where inter-element spacing $d$ is usually set as half wavelength $\lambda / 2$ to eliminate angle ambiguity. In this case, the array aperture is limited by the number of array elements. In summary, traditional algorithms need more expensive physical array elements to obtain larger array apertures and better direction of arrival estimation. In order to solve this problem, we proposed a novel scheme based on the co-prime arrays to reduce costs and improve performance. 
Figure 4 shows a uniform linear array formed by 9 elements and a co-prime array formed by 6 elements. The co-prime array in the above picture reduces the overhead of three array elements while ensuring the same array aperture and the number of reduced array elements can be expressed as $M N d-N d-M-N+1$.

\subsection{Angle ambiguity}

Co-prime array is composed of uniform linear arrays. When the spacing between elements is a multiple of half the wavelength, the angle ambiguity will occur. However, due to the property of co-prime arrays, this problem can be easily solved. The sine of both AOA and AOD can be expressed as $u_{i}$. Every angle corresponds to a map and the map of theithpath can be defined by $\Theta_{i}^{L} . L$ is the number of array element which is $M$ or $N$. Because of the periodicity of the negative exponential function, the guidance vectors of all elements in this subarray are not different. This is the reason for the ambiguity of angle.

For a certain $u_{i}, \Theta_{i}^{N}$ and $\Theta_{i}^{M}$ can be obtained respectively as the following.

$$
\begin{aligned}
& \Theta_{i}^{N}=\left\{u\left|u=u_{i}+\frac{2 n}{N}, n \in Z,-1\right| \leq u<1\right\} \\
& \Theta_{i}^{M}=\left\{u \mid u=u_{i}+\frac{2 m}{M}, m \in Z,-1 \leq u<1\right\}
\end{aligned}
$$

The property of $\Theta_{i}^{L}$ and the corresponding proof are given below.

\subsubsection{Property}

Given that $u_{i}$ and $u_{i+1}$ have the same steering vectors, $\Theta_{1}^{L}$ is equal to $\Theta_{2}^{L}$ for a subarray when the inter-element spacing is $L d$.

\subsubsection{Proof}

The steering vectors of $u_{i}$ can be defined as $a_{i}$

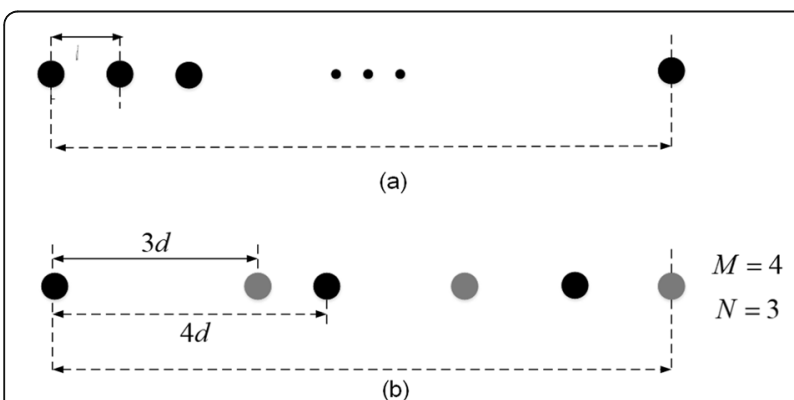

Fig. 4 Comparison of uniform and co-prime arrays. a Uniform linear array formed by 9 elements. b Co-prime array formed by 6 elements. It compares the uniform array with the non-uniform array, showing the advantages of the latter

$$
a_{i}=\left[1, e^{j \pi N u_{i}}, \cdots, e^{j \pi N(M-1) u_{i}}\right]^{T}
$$

Assuming $u_{1}$ and $u_{2}$ has the same steering vectors, then we get $a_{1}=a_{2}$ and the Eq. (7)

$$
u_{2}=u_{1}+\frac{2 \bar{n}}{L}, \bar{n} \in Z
$$

Substitute Eq. (7) into Eq. (5), we get

$$
\begin{aligned}
\Theta_{2}^{M} & =\left\{u \mid u=u_{2}+\frac{2 n}{N}\right\} \\
& =\left\{u \mid u=u_{1}+\frac{2 n}{N}+\frac{2 \bar{n}}{N}\right\} \\
& =\left\{u \mid u=u_{1}+\frac{2 n}{N}\right\} \\
& =\Theta_{1}^{M}
\end{aligned}
$$

If there is $w \in \Theta_{i}^{M}$ and $w \in \Theta_{i}^{N}$, according to Eq. (4) and Eq. (5).

$$
\left\{\begin{array}{l}
w=u_{i}+\frac{2 n}{N}, n \in Z \\
w=u_{i}+\frac{2 m}{M}, n \in Z
\end{array},-1 \leq w \leq 1 \quad \Rightarrow \quad \frac{n}{N}=\frac{m}{M}\right.
$$

There are two situations at this time shown by Eq. (10).

$$
\left\{\begin{array}{c}
\text { if } m=n=0 \quad \Rightarrow \quad v=u_{i} \\
\text { else } \quad \Rightarrow \quad \frac{n}{m}=\frac{N}{M}, \quad n \in Z, m \in Z
\end{array}\right.
$$

$M$ and $N$ are co-prime integers, so $w \geq 1$ or $w<1$; however, this is contradictory to the assumption, so

$$
\left\{u_{i}\right\}=\Theta_{i}^{M} \cap \Theta_{i}^{N} .
$$

In order to explain the above theory more vividly, the simulation results is given here. When $\mathrm{M}=5$ and $\mathrm{N}=3$, the MUSIC spectrums are depicted in Fig. 5 where we found a unique peak of coincidence and we get the actual sine $u=-0.5$. When the subarray has five elements, three peaks can be found in the spatial spectrum and when the subarray has three elements, five peaks can be found in the spatial spectrum. In fact, only the two peaks of the eight ones are meaningful, and the rest of the peak information is invalid. This phenomenon is called angle ambiguity.

\section{Proposed channel estimation scheme for co- prime arrays}

The novel channel estimation is based on partial spectral search method which can tackle the angle ambiguity.

Before introducing the proposed channel estimation scheme for co-prime arrays, we first review the channel model for mm-wave system in paper [8]. 


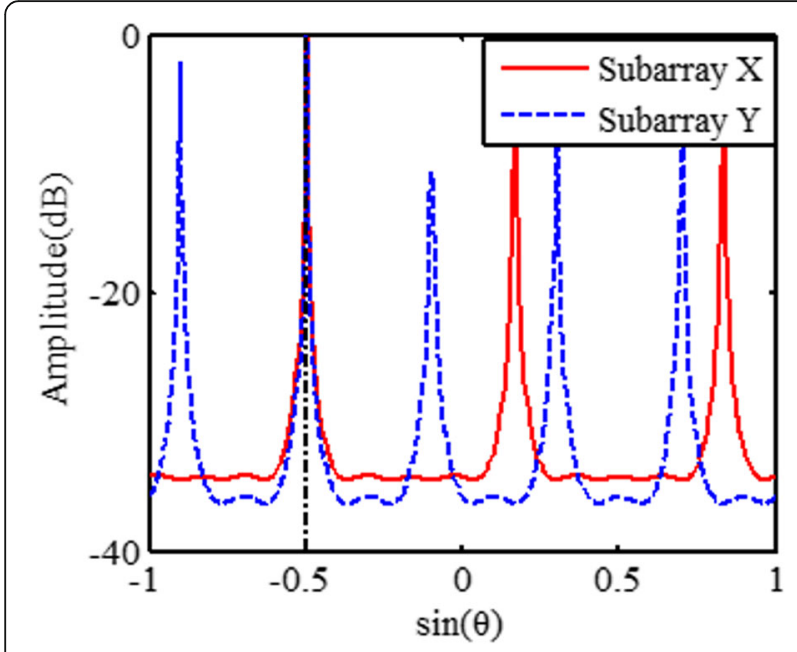

Fig. 5 MUSIC spectral of the decomposed two uniform linear arrays. The picture shows MUSIC spectrums with $M=5$ and $N=3$ are shown for the case of only one sine

\subsection{Estimation of path direction}

Supposing there are $M_{t}$ pilot signals at the transmitter where $M_{t}$ is the number of RF chains, the total transmission power is defined as $E=M_{t} P$ and the transmit power allocated equally for each pilot signal. The received signal $\mathbf{y}(q)$ is obtained during one fading block as

$$
\mathbf{y}(q)=P \mathbf{W}^{H} \mathbf{H}(q) \mathbf{F}+\mathbf{n}(q)
$$

Substituting (2) into (12), we get

$$
\mathbf{y}=P \mathbf{W}^{H} \mathbf{A}_{R}\left(\theta_{r}, \phi_{r}\right) \boldsymbol{\Lambda}_{G}(q) \mathbf{A}_{T}\left(\theta_{t}, \phi\right) \mathbf{F}+\mathbf{n}
$$

In the following, we can split a co-prime L-shaped array into two uniform linear arrays as Fig. 6 so that it can be processed separately.

The received signal model can be divided into $\mathbf{y}_{1}$ and $\mathbf{y}_{2}$.

$$
\begin{aligned}
& \mathbf{y}_{1}=P \mathbf{W}^{H} \mathbf{A}_{R 1}\left(\theta_{r}, \phi_{r}\right) \boldsymbol{\Lambda}_{G} \mathbf{A}_{T 1}^{\mathrm{T}}\left(\theta_{t}, \phi_{t}\right) \mathbf{F}+\mathbf{n} \\
& \mathbf{y}_{2}=P \mathbf{W}^{H} \mathbf{A}_{R 2}\left(\theta_{r}, \phi_{r}\right) \boldsymbol{\Lambda}_{G} \mathbf{A}_{T 2}^{\mathrm{T}}\left(\theta_{t}, \phi_{t}\right) \mathbf{F}+\mathbf{n}
\end{aligned}
$$

First, we process the signal $\mathbf{y}_{1}$ in the first array. Equation (14) can be further formulated as (16), where $\mathbf{Z}_{G T 1}$ $=\boldsymbol{\Lambda}_{G} \mathbf{A}_{T 1}^{\mathrm{T}}\left(\theta_{t}, \phi_{t}\right) \mathbf{F}$ and $\mathbf{B}_{R 1}\left(\theta_{r}, \phi_{r}\right)=P \mathbf{W}^{H} \mathbf{A}_{R 1}\left(\theta_{r}, \phi_{r}\right)$, in other words, taking $P \mathbf{W}^{H} \mathbf{A}_{R 1}\left(\theta_{r}, \phi_{r}\right)$ as a whole with the information of $\mathrm{AOA}$ and taking $\boldsymbol{\Lambda}_{G} \mathbf{A}_{T 1}^{\mathrm{T}}\left(\theta_{t}, \phi_{t}\right) \mathbf{F}$ as whole with the information of path gain.

$$
\mathbf{y}_{1}=\mathbf{B}_{R 1}\left(\theta_{r}, \phi_{r}\right) \mathbf{Z}_{G T 1}+\mathbf{n}
$$

In order to estimate the path direction, we collect the measurement vectors from the $N_{\mathrm{b}}$ blocks to compose the covariance matrices as follows.

$$
\mathbf{R}_{1}=E\left\{\mathbf{y}_{1}(q) \mathbf{y}_{1}(q)^{H}\right\}=\frac{1}{N_{\mathrm{b}}} \sum_{q=1}^{N} \mathbf{y}_{1}(q) \mathbf{y}_{1}(q)^{H}
$$

Substituting (16) into (17), the eigenvalue decomposition of $\mathbf{R}_{1}$ is expressed as Eq. (18) where $\mathbf{U}_{s}$ and $\mathbf{U}_{n}$ are signal subspace and noise subspace, respectively.

$$
\mathbf{R}_{1}=\mathbf{U}_{s} \Sigma_{s} \mathbf{U}_{s}^{H}+\mathbf{U}_{n} \Sigma_{n} \mathbf{U}_{n}^{H}
$$

Because of the orthogonality of signal subspace and noise subspace, the spatial spectrum search for the subarray 1 can be described as

$$
P_{R 1}\left(\theta_{r}, \phi_{r}\right)=\frac{1}{\mathbf{B}_{R 1}^{H}\left(\theta_{r}, \phi_{r}\right) \mathbf{U}_{n} \mathbf{U}_{n}^{H} \mathbf{B}_{R 1}\left(\theta_{r}, \phi_{r}\right)}
$$

Then, we process the signal $\mathbf{y}_{2}$ in the second array and we get Eq. (20) in the same way
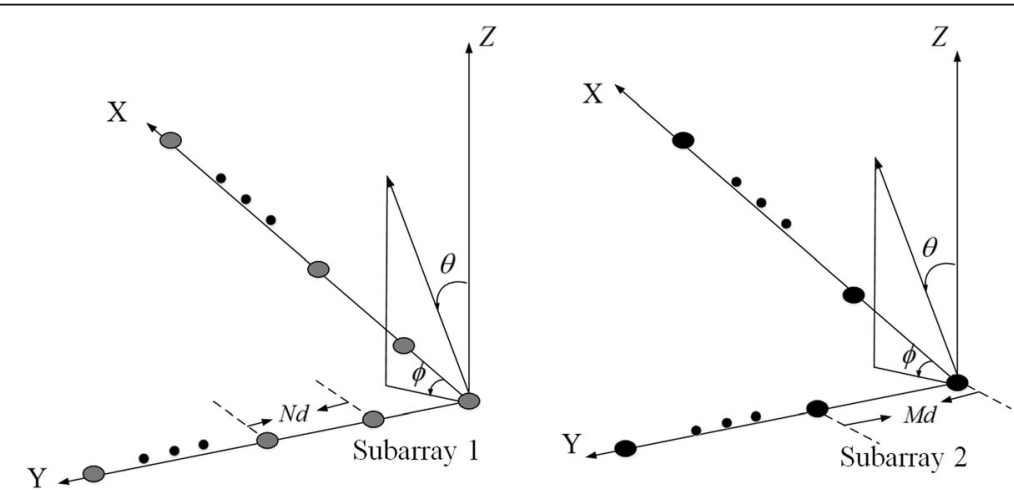

Fig. 6 The two L-shaped uniform arrays. It depicts two uniform arrays after splitting an L-shaped area array 


$$
P_{R 2}\left(\theta_{r}, \phi_{r}\right)=\frac{1}{\mathbf{B}_{R 2}^{H}\left(\theta_{r}, \phi_{r}\right) \mathbf{U}_{n} \mathbf{U}_{n}^{H} \mathbf{B}_{R 2}\left(\theta_{r}, \phi_{r}\right)}
$$

Next, we should look for the common peaks in $P_{R 1}$ and $P_{R 2}$; however, the actual element spacing is not equal to half wavelength but a multiple of $\lambda / 2$. Even if there is only one true angle value, multiple peaks will be found when performing a spatial spectrum search. Therefore, he actual two-dimensional DOA and the remaining DOA are distributed regularly over the period, based on this fact, the ambiguous 2-D DOAs can be represented by $\theta_{a}, \phi_{a}$ and the actual ones can be represented as $\theta_{c}, \phi_{c}$. According to Eq. (7), we have

$$
\begin{aligned}
& \sin \theta_{c} \cos \phi_{c}-\sin \theta_{a} \cos \phi_{a}=\frac{2 \bar{n}_{1}}{M}, \bar{n}_{1} \in(-M, M) \\
& \sin \theta_{c} \sin \phi_{c}-\sin \theta_{a} \sin \phi_{a}=\frac{2 \bar{n}_{2}}{N}, \bar{n} \in(-N, N)
\end{aligned}
$$

By performing a transformation as $\mu=\sin (\cdot)$ and $v=$ $\cos (\cdot)$, the relationship in Eq. (21) can be converted as

$$
\begin{aligned}
& \mu\left(\theta_{c}\right) v\left(\phi_{c}\right)-\mu\left(\theta_{a}\right) v\left(\phi_{a}\right)=\frac{2 \bar{n}_{1}}{M}, \bar{n}_{1} \in(-M, M) \\
& \mu\left(\theta_{c}\right) \mu\left(\phi_{c}\right)-\mu\left(\theta_{a}\right) \mu\left(\phi_{a}\right)=\frac{2 \bar{n}_{2}}{N}, \bar{n} \in(-N, N)
\end{aligned}
$$

From the above formula, we need to search for an arbitrary peak and then calculate the coordinates of other peaks based on the position of the first peak. This allows us to start searching within a small range instead of a full search, so that our new algorithm can greatly reduce the calculation range. Before the algorithm is executed, the search interval of the two arrays needs to be evenly divided and a random interval is selected to obtain the position of the peak value. Then, we can recover all the others using the relationship Eq. (22).

It just follows from the above property in Section 3.2 that each of the two subarrays will have multiple peaks. In theory, the true angle estimation is obtained by finding the positions of common peaks in the spectrum. Because the noise cannot be eliminated, there is virtually no peak that will completely coincide. So we need to compare the distance between all the peaks to find the closest ones to estimate AOA in Eq. (23) where $\left(\widehat{\theta}_{r M}\right.$, $\left.\widehat{\phi}_{r M}\right)$ and $\left(\widehat{\theta}_{r N}, \widehat{\phi}_{r N}\right)$ denote 2-d MUSIC peak for the subarray 1 and subarray 2 in Fig. 6, respectively.

$$
\widehat{\theta}_{r}=\frac{\widehat{\theta}_{r M}+\widehat{\theta}_{r N}}{2}, \widehat{\phi}_{r}=\frac{\widehat{\phi}_{r M}+\widehat{\phi}_{r N}}{2}
$$

Then, we use the same method to estimate the AOD, getting the conjugate transpose $\mathrm{y}_{1}^{H}(q)$ and $\mathrm{y}_{2}^{H}(q)$

$$
\begin{aligned}
& \mathbf{y}_{1}^{H}=\mathbf{B}_{T 1}\left(\theta_{t}, \phi_{t}\right) \mathbf{Z}_{G R 1}+\mathbf{n} \\
& \mathbf{y}_{2}^{H}=\mathbf{B}_{T 2}\left(\theta_{t}, \phi_{t}\right) \mathbf{Z}_{G R 2}+\mathbf{n}
\end{aligned}
$$

Next, we should look for the common peaks by repeat the Eq. (16) to Eq. (23) formula and we get $\widehat{\theta}_{t}$ and $\widehat{\phi}_{t}$.

\subsection{Estimation of channel}

The PSS-MUSIC method has been applied to obtain the path directions. In this section, the estimated angles can be used to obtain the path gain. We firstly calculate $\tilde{\mathbf{B}}_{R 1}$ in Eq. (16) and then uses LS method to estimate $\tilde{\mathbf{Z}}_{G T 1}$. Then the gain $\tilde{\Lambda}_{G}$ can be computed. In particular, the gain here depends on $\tilde{\mathbf{B}}_{R 1}$, because the angle change belongs to large-scale fading and the gain change belongs to small-scale fading.

$$
\begin{aligned}
\tilde{\mathbf{Z}}_{G T 1}(q) & =\left(\tilde{\mathbf{B}}_{R 1}^{H} \tilde{\mathbf{B}}_{R 1}\right)^{-1} \tilde{\mathbf{B}}_{R 1}^{H} \mathbf{y}_{1}(q), q \\
& =1,2, \cdots, N \\
\tilde{\mathbf{\Lambda}}_{G}(q) & =\tilde{\mathbf{Z}}_{G T 1}(q)\left(\tilde{\mathbf{A}}_{T 1}^{\mathrm{T}} \mathbf{F}\right)^{\dagger}
\end{aligned}
$$

Therefore, the gain is estimated according to the obtained path direction during each time block. In the end, $\tilde{\mathbf{H}}$ can be estimated as shown in Eq. (27).

$$
\tilde{\mathbf{H}}=\tilde{\mathbf{A}}_{R}\left(\theta_{r}, \phi_{r}\right) \tilde{\mathbf{\Lambda}}_{G} \tilde{\mathbf{A}}_{T}\left(\theta_{t}, \phi_{t}\right)
$$

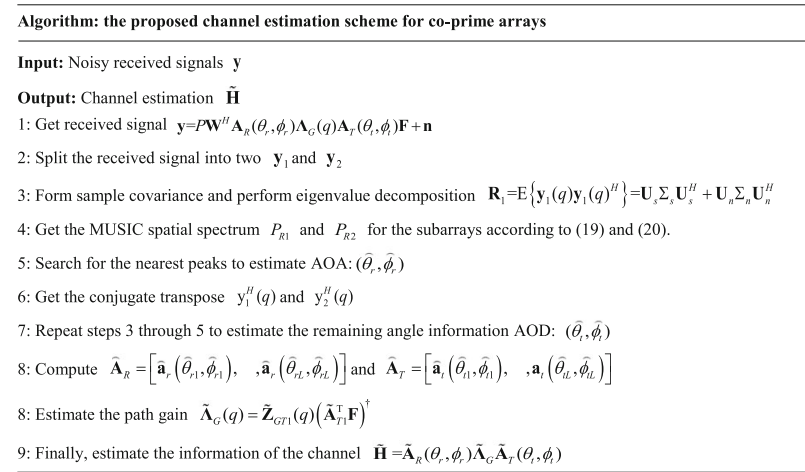

\section{Results and discussion}

In this section, numerical simulation results are provided to assess the performance of the proposed 2-d channel estimation scheme for co-prime arrays. Firstly, by comparing the performance of the same algorithm in different array antenna systems, the advantages of the coprime array compared to the uniform array are further proved in Fig. 7. Considering $2 \mathrm{~mm}$-wave MIMO communication systems with hybrid precoding are equipped with different L-shaped antenna arrays where one is a co-prime array and the other is a uniform array. The number of uniform L-shaped array elements in the $X$ axis and $Y$-axis directions is $N_{\mathrm{X}}$ - uniform and $N_{\mathrm{Y} \text { - uniform. }}$ Correspondingly, the number of co-prime arrays are $N_{\mathrm{X}}$ 


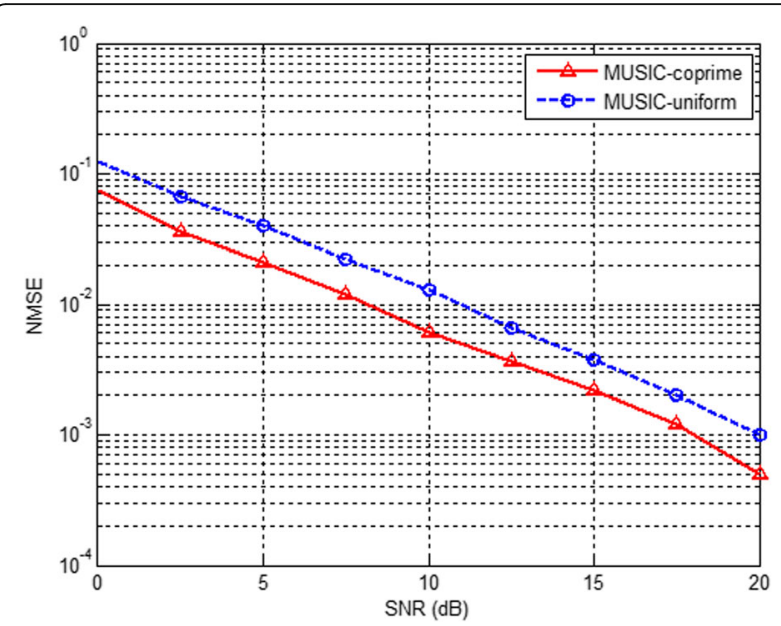

Fig. 7 The NMSE under same method versus SNR when different arrays. It compares the estimated performance of the same channel estimation method based on MUSIC algorithm in different

array systems

- coprime and $N_{Y \text {-coprime. It should be noted that in order }}$ to make the two arrays have the same aperture, the number of elements in the uniform array is more than that in the co-prime array. In order to compare the estimation performance of the proposed algorithm under two different array conditions, the remaining parameters are set to be the same. There are $M_{t} \mathrm{RF}$ chains at the transmitter and $M_{r}$ RF chains at the receiver for the two kinds of array models. The detail simulation parameters are defined as follow where the $N_{b}$ represents the number of block's pilots. The experimental parameter settings in Fig. 7 are as shown in Table 1.

Assuming that there is only one channel path, the $\mathrm{AOA}$ is $\left(17.55^{\circ}, 32.85^{\circ}\right)$ and $\mathrm{AOD}$ is $\left(19.45^{\circ}, 36.10^{\circ}\right)$. The results of the following simulation effectively show the value of the new array structure. From Fig. 7, we can see that channel estimation using a co-prime array is less complex than using a conventional uniform antenna array and the estimation accuracy is higher in the same

Table 1 Simulation parameters

\begin{tabular}{|c|c|}
\hline Parameters & Value \\
\hline$N_{X \text { - coprime }}$ & 13 \\
\hline$N_{Y-\text { coprime }}$ & 11 \\
\hline$N_{X-\text { uniform }}$ & 32 \\
\hline$N_{Y-\text { uniform }}$ & 32 \\
\hline$M_{r}$ & 4 \\
\hline$M_{t}$ & 4 \\
\hline$N_{b}$ & 60 \\
\hline$c$ & $3 \times 10^{8} \mathrm{~m} / \mathrm{s}$ \\
\hline$f_{c}$ & $3.1 \times 10^{8} \mathrm{~m} / \mathrm{s}$ \\
\hline Monte Carlo & 10000 \\
\hline
\end{tabular}

time. The normalized mean squared error (NMSE) of the channel is defined as follows.

$$
N M S E=E\left\{\|\tilde{\mathbf{H}}-\mathbf{H}\|_{F}^{2} /\left\{\|\mathbf{H}\|_{F}^{2}\right\}\right\}
$$

Through the above picture, we have proved the superiority of co-prime array. Next, we study the effect of the number of array elements on the channel estimation performance in the co-prime arrays. Set parameters $M=$ 13, $N=11$ in one array and then set $M=31, N=29$ in another array. Figure 8 shows the effect of the number of array elements on the channel estimation performance in a co-prime, we can see that the more the number of arrays, the worse the estimated performance.

The effects of different arrays on channel estimation are discussed above. The following shows how the computational complexity is reduced. Angle estimation method based on MUSIC and an improved version of PSS-MUSIC are analyzed respectively. The results are shown in Table 2. The number of array elements of two uniform arrays constituting a co-prime array is $M$ and $N$, respectively. Besides covariance matrix estimation and eigenvalue decomposition, the complexity of DOA algorithm is also affected by spectral search. The times of spectral search can be expressed as $N_{\text {search }}$, and the number of snapshots can be represented as $K$. The corresponding complexities of MUSIC are $\mathrm{O}((\mathrm{M}+\mathrm{N}-$ $\left.1)^{2} K\right), \quad O\left((M+N-1)^{3}\right)$, and $O\left(N_{\text {search }}(M+N-1)^{2}\right)$, respectively. The complexity of PSS-MUSIC in the proposed scheme is $O\left(\left(M^{2}+N^{2}\right) K\right), \quad O\left(M^{3}+N^{3}\right), \quad$ and $O\left(2 N_{\text {search }}\left(M^{2} / N+N^{2} / M\right)\right)$.

The abscissa of Fig. 9 is the number of complex elements, and the ordinate is the complex multiplication number. The parameters of the system are set as follows:

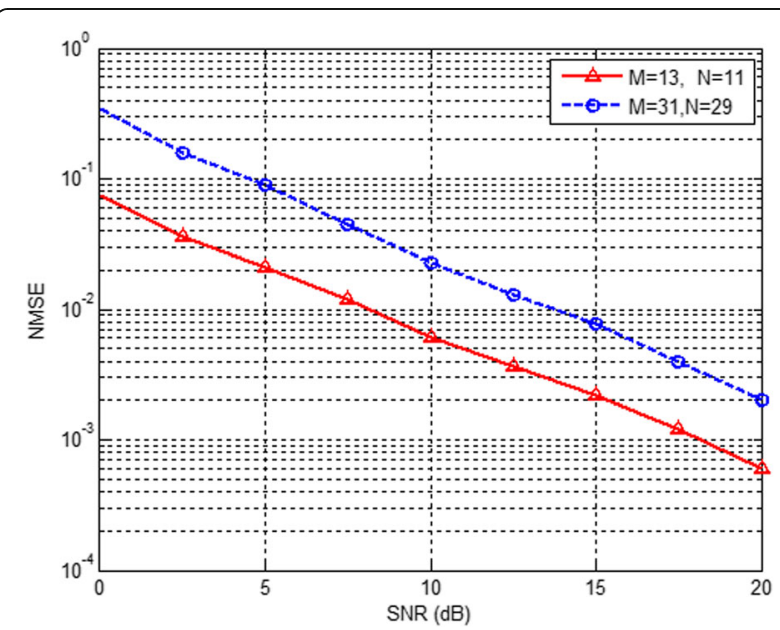

Fig. 8 The NMSE versus SNR under two co-prime with different element numbers. The figure compares the performance of channel estimation in a multi-element array with different array elements 
Table 2 Computational complexity of different methods

\begin{tabular}{ll}
\hline Algorithm & Complex multiplication \\
\hline MUSIC & $O\left((M+N-1)^{2} K+(M+N-1)^{3}+N_{\text {search }}(M+N-1)^{2}\right)$ \\
PSS-MUSIC & $O\left(\left(M^{2}+N^{2}\right) K+M^{3}+N^{3}+2 N_{\text {search }}\left(M^{2} / N+N^{2} / M\right)\right)$ \\
\hline
\end{tabular}

$K=200$ and $N_{\text {search }}=3600$. Under the condition of $M=$ 31 and $N=29$, the complex multiplications of the MUSIC algorithm reach $4.7 \times 10^{6}$ and the complex multiplications of the PSS-MUSIC are $4.0 \times 10^{6}$. We can get that the computational complexity of the latter is $62 \%$ of the former, which is the basis for our proposed algorithm to reduce computational complexity. It is expected that when the number of array elements is large, the PSS-MUSIC based channel estimation will have lower computational complexity. Therefore, the proposed estimation scheme can realize faster channel estimation.

Before demonstrating the new method channel estimation performance, we first verify the accuracy of the angular accuracy estimation accuracy of the new algorithm. The MSE of the estimated path angles are defined as

$$
M S E=E\left\{\left(\phi_{t}-\hat{\phi}_{t}\right)^{2}+\left(\theta_{t}-\hat{\theta}_{t}\right)^{2}+\left(\phi_{r}-\hat{\phi}_{r}\right)^{2}+\left(\theta_{r}-\hat{\theta}_{r}\right)^{2}\right\}
$$

Figure 10 depicts the MSE versus SNR via 10000 Monte Carlo simulations and shows that the PSS-based angle estimation performs better than the traditional TSS-MUSIC method

We set $M=7$ and $N=5$ in the above simulation system where one channel path is assumed, and the AOA is $\left(7.95^{\circ}, 10.85^{\circ}\right)$ and $\mathrm{AOD}$ is $\left(11.45^{\circ}, 16.10^{\circ}\right)$. The search

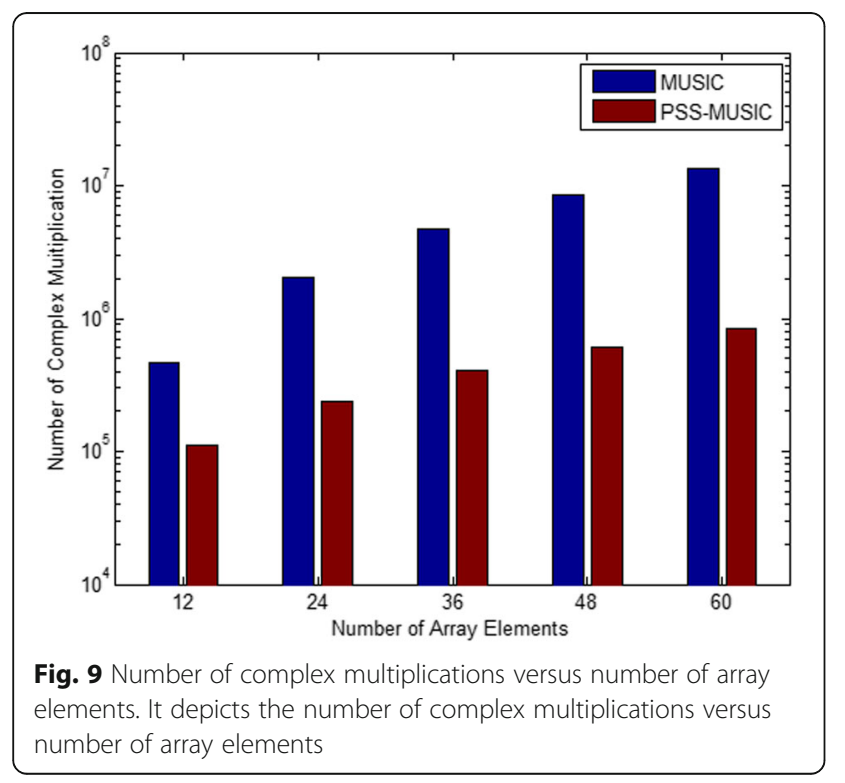

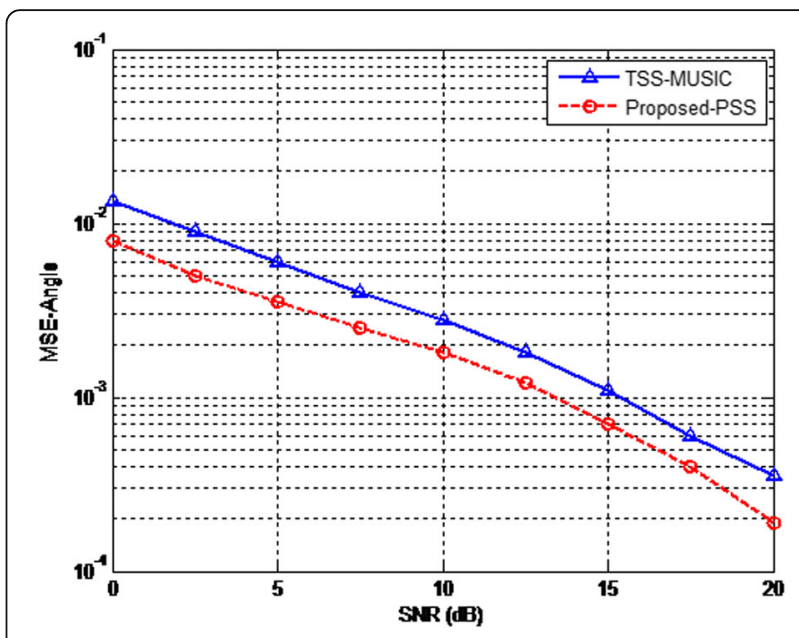

Fig. 10 MSE of angles estimation versus SNR. It plots the MSE versus SNR via 10000 Monte Carlo simulations and shows that the PSSbased angle estimation performs better than the traditional TSS-MUSIC method

time depends not only on the size of the search grid, but also on the range of search angles. It is known from the previous analysis that instead of total spectral search, the channel estimation scheme based on the PSS algorithm can search over a limited area. In order to narrow the difference in the times of searching between the two methods, the searching grid for MUSIC is set to $0.05^{\circ}$ and the one for PSS-MUSIC is set to $0.01^{\circ}$.

Figure 11 shows that the proposed channel estimation performs better than the method based on TSS-MUSIC. One channel path is assumed as above Fig. 10 where $M=7$ and $N=5$. For the same reason as above, the searching grid for MUSIC is set to $0.05^{\circ}$ and the one for PSS-MUSIC is set to $0.01^{\circ}$.

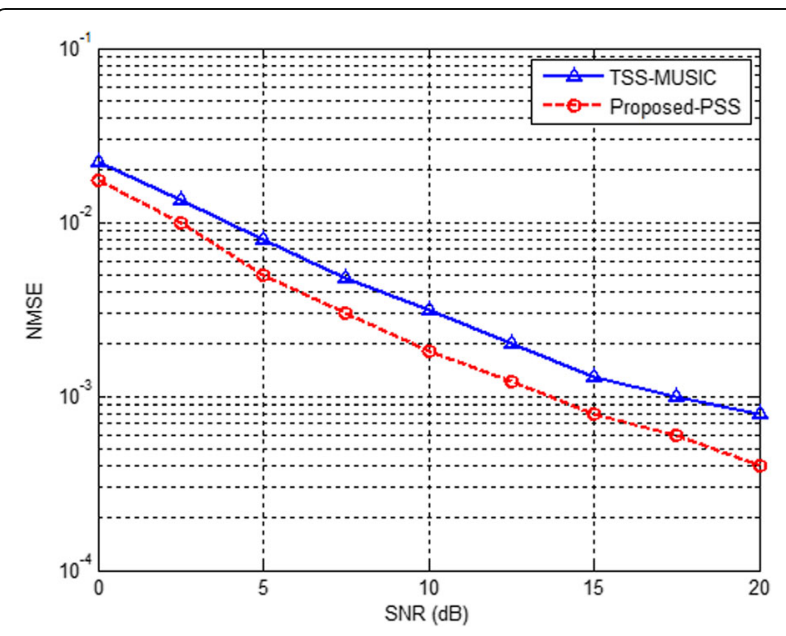

Fig. 11 The NMSE versus SNR. The picture shows that the proposed channel estimation performs better than the method based on TSSMUSIC $M=7$ and $N=5$ 


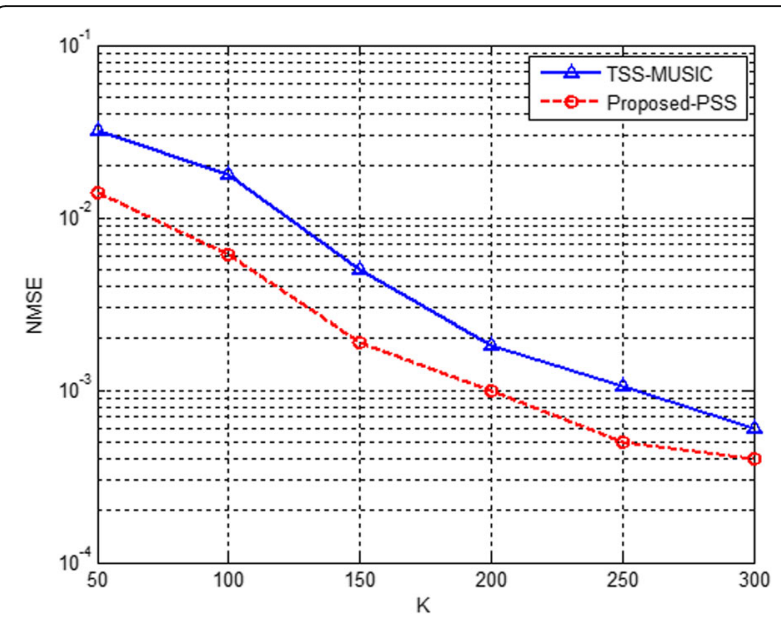

Fig. 12 The NMSE versus number of block's pilots. The figure plots the reverses number of block's pilots via 10000 Monte Carlo simulations with $\mathrm{SNR}=10 \mathrm{~dB}$

Figure 12 plots the reverses number of block's pilots via 10000 Monte Carlo simulations with $\mathrm{SNR}=10 \mathrm{~dB}$. From the figure, we can see that the performance of the two channel estimation methods gets better with the increase of the number of block's pilots, but overall, the performance of the new algorithm is always better than the old algorithm. It illustrates the superiority of the proposed scheme from another aspect.

\section{Conclusions}

In this paper, we proposed a channel estimation scheme for co-prime arrays and the proposed scheme is based on the PSS-MUSIC method. The simulation results show that the novel method can effectively estimate the channel state information in co-prime arrays while greatly reducing the computational complexity. However, we have to admit that our research also has a limitation, that is, as the number of channel paths increases, spectrum searching between different paths may be confused. For future work, it would be interesting to work on how to solve this problem which may help to further improve the accuracy of channel estimation co-prime.

\section{Abbreviations}

2-d: Two-dimensional; AOA: Angle of arrival; AOD: Angle of departure; DOA: Direction-of-arrival; mm-wave: Millimeter wave; PSS-MUSIC: Partial spectral search MUSIC method; TSS: Total spectral search; ULAs: Uniform linear arrays

\section{Acknowledgements}

The authors acknowledged the three anonymous reviewers and editors for their efforts in constructive and generous feedback.

\section{Authors' contributions}

$\mathrm{SL}$ contributed to the conception and design of the study. GC is the main author of the current paper. $L$ and HW commented on the work. All authors read and approved the final manuscript.

\section{Funding}

This work was supported by the National Nature Science Funding of China (NSFC): 61401407 and the Fundamental Research Funds for the Central Universities.

\section{Availability of data and materials}

Not applicable

\section{Competing interests}

The authors declare that they have no competing interests.

\section{Author details}

${ }^{1}$ School of Information and Telecommunication Engineering, Communication University of China, Beijing 100024, People's Republic of China. ${ }^{2}$ Department of Electrical Engineering and Computer Science, York University, Toronto, Canada.

Received: 30 October 2019 Accepted: 31 December 2019

Published online: 13 January 2020

\section{References}

1. Z. Xiao, P. Xia, X.G. Xia, Channel estimation and hybrid precoding for millimeter-wave MIMO systems: A low-complexity overall solution. IEEE Access 5(99), 16100-16110 (2017)

2. S. Rangan, T. S. Rappaport and E. Erkip, Millimeter wave cellular wireless networks: potentials and challenges. Proc IEEE, 102(3), 366-385, March (2014)

3. Y. Sun, G. Feng, S. Qin, S. Sun, Cell association with user behavior awareness in heterogeneous cellular networks. IEEE Trans Vehicular Technol 67(5), 4589-4601 (2018)

4. C Hu, J H Zhang, 2017 IEEE 6th Global Conference on Consumer Electronics (GCCE). Hybrid precoding design for adaptive subarrays in millimeter-wave MIMO (Nagoya, Japan, 2017), pp. 24-27

5. Y Sun, L Zhang, G Feng, B Yang, B Cao, and Muhammad Ali Imran, Blockchain-enabled wireless internet of things: Performance analysis and optimal communication node deployment, IEEE Internet Things J, 57915802 (2019)

6. J Mo, P Schniter, N G Prelcic, and R W Heath, 2014 48th Asilomar Conference on Signals. Systems and computers channel estimation in millimeter wave MIMO systems with one-bit quantization (USA, 2014), pp. 1058-6393

7. M D Mueck, I Karls, R Arefi, 2014 IEEE Globecom Workshops (GC Wkshps). Global standards enabling a 5th generation communications system architecture vision (USA,2014), pp. 571-575

8. Y. M. Tsang, A. S. Y. Poon and S. Addepalli, IEEE Global Telecommunications Conference. Coding the beams: Improving beamforming training in mmWave communication system (Kathmandu, Nepal, 2011), pp. 1-6

9. A. Alkhateeb, O.E. Ayach, G. Leus, Channel estimation and hybrid precoding for millimeter wave cellular systems. IEEE J Selected Topics Signal Processing 8(5), 831-846 (2014)

10. D. Zhu, J. Choi, R.W. Heath, Auxiliary beam pair enabled AOD and AOA estimation in closed-loop large-scale millimeter-wave MIMO systems. IEEE Trans Wireless Commun 16(7), 4770-4785 (2017)

11. J. Lee, G. T. Gill and Y. H. Lee, IEEE Global Communications Conference. Exploiting spatial sparsity for estimating channels of hybrid MIMO systems in millimeter wave communications (Austin, TX, USA, 2014), pp. 3326-3331

12. O.E. Ayach, S. Rajagopal, S. Abu-Surra, Spatially sparse precoding in millimeter wave MIMO systems. IEEE Trans Wireless Commun 13(3), 1499 $1513(2014)$

13. Y.J. Guo, X. Huang, V. Dyadyuk, A hybrid adaptive antenna array for longrange mm-wave communications. IEEE Antennas Propagation Mag 54(2), 271-282 (2012)

14. A. Liu, V. Lau, Phase only RF precoding for Massive MIMO systems with limited RF chains. IEEE Trans Signal Process 62(17), 4505-4515 (2014)

15. J. Lee, G.T. Gil, Y.H. Lee, Channel estimation via orthogonal matching pursuit for hybrid MIMO systems in millimeter wave communications. IEEE Trans Commun 64(6), 2370-2386 (2016)

16. Z. Marzi, D. Ramasamy, U. Madhow, Compressive channel estimation and tracking for large arrays in $\mathrm{mm}$-wave picocells. IEEE J Select Topics Signal Process 10(3), 514-527 (2016) 
17. C. Hu, L.L. Dai, T. Mir, Z. Gao, Super-resolution channel estimation for mmwave massive MIMO with hybrid precoding. IEEE Trans Vehicular Technol 67(9), 8954-8958 (2018)

18. X. Wei, C. Hu, L. Dai, Knowledge-aided deep learning for channel estimation in millimeter-wave massive MIMO systems. IEEE Wireless Commun Lett 7(5), 852-855 (2019)

19. Y. Sun, G. Feng, S. Qin, Y.-C. Liang, The SMART handoff policy for millimeter wave heterogeneous cellular networks. IEEE Trans Mobile Comput 17(6), 1456-1468 (2018)

20. T. S. Rappaport, R. W. Heath and R. C. Daniels, Millimeter wave wireless communications. Upper Saddle River, NJ, USA: Prentice-Hall (2014)

21. W. Zhang, W. Liu, J. Wang, S. Wu, Joint transmission and reception diversity smoothing for direction finding of coherent targets in MIMO radar. IEEE J Select Topics Signal Process 8(1), 115-124 (2014)

22. R. Xie, Z. Liu, J.K. Wu, Direction finding with automatic pairing for bistatic MIMO radar. Signal Process 92(1), 198-203 (2012)

23. S.F. Li, G.J. Cao, L.B. Jin, Two-dimensional channel estimation for millimeterwave MIMO systems with hybrid precoding. J Internet Technol 19(6), 1871$1877(2018)$

24. P.P. Vaidyanathan, P. Pal, Sparse sensing with co-prime samplers and arrays. IEEE Trans Signal Process 59(2), 573-586 (2011)

25. P.P. Vaidyanathan, P. Pal, Theory of sparse coprime sensing in multiple dimensions. IEEE Trans Signal Process 59(8), 3592-3608 (2011)

26. G. Qin, Y.D. Zhang, M. Amin, DOA estimation exploiting moving dilated nested arrays. IEEE Signal Process Lett 26(3), 490-494 (2019)

27. A M Elbir. L-shaped coprime array structures for DOA estimation. Multidimensional Systems and Signal Processing, 1-15 (2019).

28. R.O. Schmidt, Multiple emitter location and signal parameter estimation. IEEE Trans Antennas Propagation 34(3), 276-280 (1986)

29. R. Roy, T. Kailath, Esprit-estimation of signal parameters via rotational invariance techniques. IEEE Trans Acoustics Speech Signal Process 37(7), 984-995 (1989)

30. B.D. Rao, K.V.S. Hari, Performance analysis of root-music. IEEE Trans Acoustics Speech Signal Process 37(12), 1939-1949 (1989)

31. C. Zhou, Z. Shi, Y. Gu, and X. Shen, 2013 International Conference on Wireless Communications and Signal Processing. DECOM: DOA estimation with combined MUSIC for coprime array (Hangzhou, China, 2013), pp. 24-26

32. F. Sun, P. Lan, B. Gao, Partial spectral search-based DOA estimation method for co-prime linear arrays. Electron Lett 51(24), 2053-2055 (2015)

33. F. Sun, B. Gao, L. Chen, P. Lan, A low-complexity ESPRIT-based DOA estimation method for co-prime linear arrays. Sensors 16(9), 1367 (2016)

34. D. Zhang, Y.S. Zhang, G.M. Zheng, C.Q. Feng, J. Tang, Improved DOA estimation algorithm for co-prime linear arrays using root-MUSIC algorithm. Electronics Letters. 53(18), 1277-1279 (2017)

\section{Publisher's Note}

Springer Nature remains neutral with regard to jurisdictional claims in published maps and institutional affiliations.

\section{Submit your manuscript to a SpringerOpen ${ }^{\circ}$ journal and benefit from:}

- Convenient online submission

- Rigorous peer review

- Open access: articles freely available online

- High visibility within the field

- Retaining the copyright to your article

Submit your next manuscript at $\boldsymbol{\nabla}$ springeropen.com 